\title{
A Review of Sensor Node in Wireless Sensor Networks
}

\author{
Jobanputra Paresh Ashokkumar1, Prof. Arun Jhapate ${ }^{2}$ \\ ${ }^{1} \mathrm{PG}$ Scholar, ${ }^{2}$ Associate Professor \\ 1,2Department of CSE, Sagar Institute of Research \& Technology, Bhopal, Madhya Pradesh, India
}

\begin{abstract}
How to cite this paper: Jobanputra Paresh Ashokkumar | Prof. Arun Jhapate "A Review of Sensor Node in Wireless Sensor Networks" Published in International Journal of Trend in Scientific Research and Development (ijtsrd), ISSN: 24566470, Volume-3 | Issue-4, June 2019, pp.124-127, URL: https://www.ijtsrd.c om/papers/ijtsrd23 620.pdf

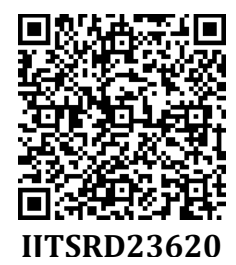

Copyright (C) 2019 by author(s) and International Journal of Trend in Scientific Research and Development Journal. This is an Open Access article distributed under the terms of the Creative Commons

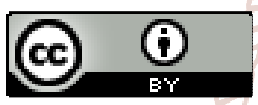
Attribution License (CC BY 4.0) (http://creativecommons.org/licenses/ by/4.0)

\section{INTRODUCTION}

A wireless sensor network is collections of a bulky amount of sensor nodes, which are closely deployed within the network area. Since the nodes are frequently inaccessible, the life of a wireless sensor network depends on the lifetime of the energy resources of the sensor nodes. Energy is also a limited resource due to the dimensions restrictions [1]. Energy efficient announcement is very important to extend the sensor network lifetime. In recent times, different routing protocols have been reported for wireless sensor networks. Many protocols apply single optimal route in support of data transmission. The optimal route is selected based on the metrics, such as minimum hop, high remaining energy, minimum broadcast cost etc. to route the data [2-5]. Though the single-route approach is easy and scalable, but choosing an optimal route and transmitting the data throughout that route may not increase the sensor network lifetime [6]. With multipath route routing in sensor networks can assist in efficient energy procedure, by regularly distributing the traffic load over the sensor network, thus expanding the sensor network lifetime. Routing is very challenging in wireless sensor networks. One of the most difficulties that involve the sensor network lifetime refers to sensor nodes in the neighborhood of the sensor sink, whose activity effect a high traffic on this sequence of sensor nodes. An additional process for the sensor sink neighborhood difficulty is to offer several of the sensor network fundamentals with mobile ability [7].
In bio-inspired techniques, in recent times have been added to the optimization techniques a vital class since they can optimize the path creation stage. Bio-inspired routing protocols which are considered based on creature sensory scheme try to create the shortest route from the source node to the sink node so that it can preserve high energy.

\section{RELATED WOR}

In 2018 [1], Internet of thing (IoT) is not only a promising research topic but also a blooming industrial trend. Although the basic idea is to bring things or objects into the Internet, there are various approaches because an IoT system is highly application oriented. In addition to the design of a customized hardware for range extension, a new synchronization scheme and a burst transmission feature are also presented to boost the network capacity and reduce the energy waste. As a result, the proposed platform can fulfill the high throughput requirement for high-rate applications and the requirement of long battery life for lowrate applications at the same time. We have developed a test bed in our campus to validate the proposed system.

In 2018 [2], In wireless sensors networks, the sensor nodes are densely deployed. Owing to this excessive deployment of sensor nodes, each target is covered by multiple sensors at a time. To prolong the network lifetime, the authors can 
schedule the sensor activity in such a way that only a subset of sensor nodes, called cover set, is sufficient enough to cover all the targets. In this study, they propose an energyefficient scheduling algorithm based on learning automata for target coverage problem. The learning automata-based technique helps a sensor node to select its appropriate state (either active or sleep). To prove the effectiveness of their proposed scheduling method, they conduct a detailed set of simulations and compare the performance of their algorithm with the existing algorithms.

In 2018 [3], This work presents a combined energy-efficient medium access control (MAC) and routing protocol for largescale wireless sensor networks that aims to minimize energy consumption and prolong the network lifetime. The proposed communication framework employs the following measures to enhance the network energy efficiency. Firstly, it provides an adaptive intra-cluster schedule to arbitrate media access of sensor nodes within a cluster, minimizing idle listening on sensor nodes, leading to improved energy performance. Secondly, it proposes an on-demand source cross-layer routing protocol ensuring selection of best routes based on energy level and channel quality indicator for the multi-hop inter-cluster data transmission. Lastly, an unequal cluster size technique based on cluster head residual energy and distance away from the base station is utilized. This technique balances the energy among clusters and avoids early network partitioning. This work further presents the analytical performance model for energy consumption and delay of the proposed communication framework. The performance measures used for evaluation are energy consumption, delay, and network lifetime. The results indicate that combining routing and MAC schemes conserves energy better than utilizing MAC scheme alone.

In 2017 [4], 3-axis accelerometer is a widely adopted device for applications such as fall detection of the elders, kinematic analysis, building/bridge/landslide monitoring, and so on. To retrieve the sensing data in real time, one may use a wireless module to forward those data to a gateway or server. For outdoor applications, Zigbee/IEEE 802.15.4 technology is often the candidate because it is cost effective and easy to be purchased in the market. However, transmission range, energy consumption and network capacity are key factors in designing an outdoor system.

In 2016 [5], Time-synchronized channel hopping (TSCH) is currently the most efficient solution for collision-free, interference avoiding communications in ad hoc wireless networks, such as wireless sensor networks, vehicular networks, and networks of robots or drones. However, all variants of TSCH require some form of centralized coordination to maintain the time-frequency slotting mechanism. This leads to slow convergence to steady state and moderate time-frequency slot utilization, especially under node churn or mobility. We propose decentralized time synchronized channel swapping (DT-SCS), a novel protocol for medium access control (MAC) in ad hoc wireless networks. Under the proposed protocol, nodes first converge to synchronous beacon packet transmissions across all available channels at the physical layer, with balanced number of nodes in each channel.

In 2016 [6], We have witnessed the Fixed Internet emerging with virtually every computer being connected today; we are currently witnessing the emergence of the Mobile Internet with the exponential explosion of smart phones, tablets and net-books. However, both will be dwarfed by the anticipated emergence of the Internet of Things (IoT), in which everyday objects are able to connect to the Internet, tweet or be queried. Whilst the impact onto economies and societies around the world is undisputed, the technologies facilitating such a ubiquitous connectivity have struggled so far and only recently commenced to take shape. To this end, this paper introduces in a timely manner the cornerstones of a technically and commercially viable IoT which includes a detailed discussion on the particular standard of choice at each protocol layer. This stack is shown to meet the important criteria of power-efficiency, reliability and Internet connectivity. Industrial applications have been the early adopters of this stack, which has become the de-facto standard, thereby bootstraping early IoT developments. Corroborated throughout this paper and by emerging industry alliances, we believe that a standardized approach, using latest developments in the IEEE 802.15.4 and IETF working groups, is the only way forward.

In 2016 [7], The main concern in Wireless Sensor Networks is how to handle with their limited energy resources. The performance of Wireless Sensor Networks strongly depends on their lifetime. As a result, Dynamic Power Management approaches with the purpose of reduction of energy consumption in sensor nodes, after deployment and designing of the network. Recently, there have been a strong interest to use intelligent tools especially Neural Networks in energy efficient approaches of Wireless Sensor Networks, due to their simple parallel distributed computation, distributed storage, data robustness, auto classification of sensor nodes and sensor reading. This paper presents a new centralized adaptive Energy Based Clustering protocol through the application of Self organizing map neural networks (called EBC-S) which can cluster sensor nodes, based on multi parameters; energy level and coordinates of sensor nodes. We applied some maximum energy nodes as weights of SOM map units; so that the nodes with higher energy attract the nearest nodes with lower energy levels. Therefore, formed clusters may not necessarily contain adjacent nodes. The new algorithm enables us to form energy balanced clusters and equally distribute energy consumption. Simulation results and comparison with previous protocols ( LEACH and LEA2C) prove that our new algorithm is able to extend the lifetime of the network.

In 2016 [8], The increasing complexity of Wireless Sensor Networks (WSNs) is leading towards the deployment of complex networked systems and the optimal design of WSNs can be a very difficult task because several constraints and requirements must be considered, among all the power consumption. This paper proposes a novel fuzzy logic based mechanism that according to the battery level and to the ratio of Throughput to Workload determines the sleeping time of sensor devices in a Wireless Sensor Network for environmental monitoring based on the IEEE 802.15.4 protocol. The main aim here is to find an effective solution that achieves the target while avoiding complex and computationally expensive solutions, which would not be appropriate for the problem at hand and would impair the practical applicability of the approach in real scenarios. The results of several real test-bed scenarios show that the proposed system outperforms other solutions, significantly reducing the whole power consumption while maintaining good performance in terms of the ratio of throughput to 
workload. An implementation on off-the-shelf devices proves that the proposed controller does not require powerful hardware and can be easily implemented on a low-cost device, thus paving the way for extensive usage in practice.

\section{DISCUSSION}

Complex networks are a widespread tool for the modeling of complex systems, they are used in many domains, including biology, communication, sociology, physics, economy, etc. [1]. In the recent years, a whole arsenal of measures was defined to study them. More interestingly for us, they have been intensively used to study computer networks, especially the Internet. Various methods were used to retrieve the Internet structure, focusing on different granularities such as the inter-domain [2-5] and router [4,5] levels. Using such a topological approach to study computer networks leads to a better understanding of the network structure, which in turn brings multiple benefits. First, improving the models used to provide simulation data, resulting in more realistic testing conditions $[3,5,6]$. All the tools specifically developed for the network can therefore be assessed more accurately. Moreover, it is also possible to consider the network structure from a dynamic perspective. By studying the evolution of the topology, one can derive predictive models [7]. A better understanding of the network structure also allows designing better management methods and tools [6]. Finally, by taking advantage of this knowledge, tone can improve communication protocols by adapting them to the network structure [2,3]. Stemming from the fact that WSNs belong to the class of computer networks, such a topological approach seems relevant to improve the tools and technologies related to them. Several works already applied complex network research tools to WSNs to better understand their dynamics and enhance their performance $[8,9]$. Moreover, in the case of WSNs, topological measures are particularly interesting because they allow comparing one network at different time steps, or several distinct networks. This makes possible assessing the effect of the deployment (spatial distribution, number of sinks, etc.) and operational (communication protocol, type of sensor, etc.) parameters on WSN-specific features, such as the network life time. However, from the topological point of view, WSNs may present considerable differences from mainstream computer networks, particularly the Internet: their temporal scale is much smaller, they shrink instead of growing, and they are structured around certain nodes with specific properties, i.e. the sinks. For this reason, topological measures for the assessment of WSNs have to be chosen with care, as the classical measures may not be directly applicable. In this article, we try to answer this question by reviewing the main measures used in previous works to study the Internet, and discussing their suitability to the analysis of WSNs. For the cases where the studied measure turns out to be inappropriate, we define alternatives and explain the intuition behind them.

\section{CONCLUSION AND FUTURE WORKS}

In this article, we described the different tools one can use to analyze the topology of WSNs. Our contribution is three-fold. First, we performed a review of the most popular measures used in existing studies regarding the Internet. We discussed their appropriateness to the study of WSNs, and pointed out their limitations in this context. Second, we defined new, WSN-specific measures aiming at solving these limitations, and explained how they could be used. Finally, we simulated some WSN activity thanks to NS2 in order to illustrate the relevance of both retained classic measures and newly proposed ones. Taking advantage of this data, we explained how their values can be interpreted to characterize the evolution of a WSN. We focused on five classes of measures. For those relative to the network size, we proposed to distinguish the live part of the WSN from the disconnected sensors. We showed how one could use the existing degreebased measures to identify critical points in the evolution of WSNs, which are characterized by a very dynamic nature. We introduced the notion of sink-distance, in order to adapt the existing distance-based measures (diameter, average distance, distance distribution, etc.) to WSNs. We defined the related notion of sink-between's in order to extend the centrality-based measures traditionally used to study the Internet. Finally, we applied transitivity-based measures to highlight a very uncommon trait of the studied WSN: its transitivity stays constant despite the fact it never stops losing nodes and links during the simulation. This work can be extended in several ways. First, for space matters, we could not be exhaustive regarding the reviewed topological properties. For instance, other centrality measures exist and are used to characterize computer networks [4]. More importantly, we had to ignore the macroscopic structure of the network, which is nevertheless of great interest. It can be studied by considering the presence of strongly connected components (i.e. maximal sub graphs in which all nodes are interconnected through directed paths) or communities. Finally we did not consider the transitivity (a.k.a. clustering coefficient), which is also a very important topological measure. Another interesting aspect concerns the measures we defined. As we stated before, although informative, traditional measures are not always relevant to study WSNs, due to their specific features, which is why we introduced our own ones. Consequently, these could be applied to networks representing different real-world systems, provided those are similar enough to WSNs. Let us consider, for instance, an egocentric social network. It is extracted by selecting first a person of interest as the center of the network, and adding its direct and indirect acquaintances, until some radius limit is reached. In this context, the sink-distance and sink-between would be of great interest to characterize acquaintances relatively to the person of interest.

\section{REFERENCES}

[1]. Yaw-Wen Kuo, Cho-Long Li, Jheng-Han Jhang and Sam Lin, "Design of a wireless sensor network based IoT platform for wide area and heterogeneous applications", IEEE Sensors Journal, 2018.

[2]. Manju, Satish Chand, Bijender Kumar, "Target coverage heuristic based on learning automata in wireless sensor networks", IET Wirel. Sens. Syst., 2018.

[3]. Maria Sefuba, Tom Walingo, "Energy-efficient medium access control and routing protocol for multihop wireless sensor networks", IET Wireless Sensor Systems, 2018.

[4]. Yaw-Wen Kuo and Cho-Long Li, "Design of a long range wireless 3-axis accelerometer module for environmental monitoring", IEEE Journal of WSN, 2017.

[5]. Arvind Kumar, Damanpreet Singh, "Importance of Energy in Wireless Sensor Networks: A Survey", An International Journal of Engineering Sciences, Vol. 17, 2016. 
[6]. George Smart, Nikos Deligiannis, Rosario Surace, Valeria Loscri, Giancarlo Fortino and Yiannis Andreopoulos, "Decentralized Time-Synchronized Channel Swapping for Ad Hoc Wireless Networks", IEEE Transaction of Wireless Network, 2016.

[7]. Maria Rita Palattella, Nicola Accettura, Xavier Vilajosana, Thomas Watteyne, Luigi Alfredo Grieco, Gennaro Boggia and Mischa Dohler, "Standardized Protocol Stack For The Internet Of (Important) Things", IEEE Conference of IoT, 2016.

[8]. Mohammad Ali Azimi kashani and Hassan Ziafat, "A method for Reduction of Energy Consumption in Wireless Sensor Network with using Neural Networks", IEEE Conference of Wireless Sensor Network, 2016.

[9]. Giovanni Pau, "Power Consumption Reduction for Wireless Sensor Networks Using A Fuzzy Approach", International Journal of Engineering and Technology Innovation, vol. 6, no. 1, 2016.

[10]. Junaid Ahmed Khan, Hassaan Khaliq Qureshi and Adnan Iqbal "Energy management in Wireless Sensor Networks: A survey", https://hal.archivesouvertes.fr/hal-01283728, 2015.
[11]. Keshav Sood, Shui Yu and Yong Xiang, "Software Defined Wireless Networking Opportunities and Challenges for Internet of Things: A Review", IEEE internet of things journal, 2015.

[12]. Ala Al-Fuqaha, Mohsen Guizani, Mehdi Mohammadi, Mohammed Aledhari and Moussa Ayyash, "Internet of Things: A Survey on Enabling Technologies, Protocols, and Applications", IEEE communication surveys \& tutorials, vol. 17, no. 4, fourth quarter 2015.

[13]. Antonio Guerrieri, Giancarlo Fortino and Wilma Russo, "An Evaluation Framework for Buildings-oriented Wireless Sensor Networks", 14th IEEE/ACM International Symposium on Cluster, Cloud and Grid Computing, 2014

[14]. B. Molina, C.E. Palau, G. Fortino, A. Guerrieri, C. Savaglio, "Empowering smart cities through interoperable Sensor Network Enablers", IEEE International Conference on Systems, Man, and Cybernetics, 2014

[15]. Mihai T. Lazarescu, "Design of a WSN Platform for Long-Term Environmental Monitoring for IoT Applications", IEEE journal on emerging and selected topics in circuits and systems, vol. 3, no. 1, march 2013

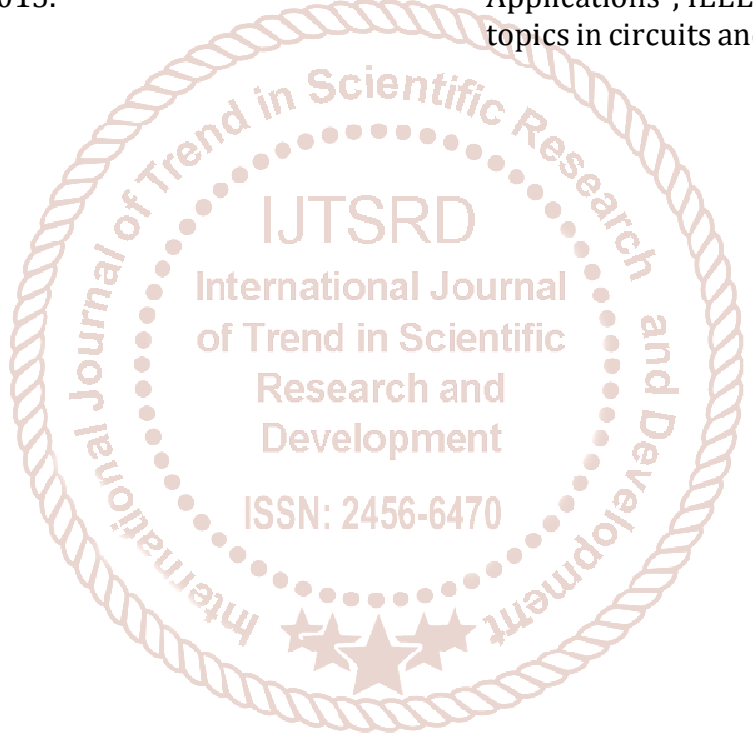

